\title{
High waves in Draupner seas-Part 1: numerical simulations and characterization of the seas
}

\author{
E. van Groesen ${ }^{1,2} \cdot$ P. Turnip ${ }^{1} \cdot$ R. Kurnia ${ }^{1,2}$
}

Received: 10 March 2017 / Accepted: 26 June 2017 / Published online: 20 July 2017

(C) The Author(s) 2017. This article is an open access publication

\begin{abstract}
Extreme waves are studied in numerical simulations of the so-called Draupner seas that resemble the wave situation near the observation area of the Draupner wave, an iconic example of a freak, rogue wave. Recent new meteorological insights describe these seas as a substantial wind-generated wave system accompanied by two low-frequency lobes. With the significant wave height $H_{\mathrm{s}}=$ $12 \mathrm{~m}$ above a depth of $70 \mathrm{~m}$ and the wide directional spreading over $120^{\circ}$ as design information, results are presented of simulations of phase resolved waves. Quantitative data are derived from 8000 waves over an area of $15 \mathrm{~km}^{2}$. Very high waves with crest heights exceeding $1.5 H_{\mathrm{s}}$ occur in average in 20 min timespan over an area of $0.8 \mathrm{~km}^{2}$. Details will be given for an isolated freak wave and a sequence of 3 freak crest heights in a group of 2 high waves. In Part 2, van Groesen and Wijaya (J Ocean Eng Mar Energy, 2017), it will be shown that $60 \mathrm{~s}$ before their appearance freak waves can be predicted from radar images on board of a ship that scans the surrounding area over a distance of $2 \mathrm{~km}$.
\end{abstract}

Keywords Freak waves - Draupner seas - Elevation exceedance $\cdot$ Multi-directional wave influx $\cdot$ Radar observation

\footnotetext{
$凶$ E. van Groesen

groesen@labmath-indonesia.org

P. Turnip

p.turnip@labmath-indonesia.org

R. Kurnia

r.kurnia@labmath-indonesia.org

1 LabMath-Indonesia, Bandung, Indonesia

2 University of Twente, Enschede, The Netherlands
}

\section{Introduction}

The study of multi-directional waves is an area of active research, in particular the study of extreme waves, also called freak or rogues waves, see for instance the overviews of Kharif and Pelinovsky (2003), Dysthe et al. (2008), and Kharif et al. (2009). In this paper, results will be presented of numerical simulations of waves in high random seas with a very wide spreading of directions. Specifically, we study what will be called Draupner seas, because the seas are constructed from properties that are known of the sea at the place and time of the measurement of the Draupner wave, Haver (1995). Use is made of new findings that are based on more accurate atmospheric simulations by Cavaleri et al. (2016) about the wave circumstances in a neighbourhood at the time of the observation of the Draupner wave. The present understanding is that under the influence of a polar low-pressure disturbance that was not taken into account previously, the severe storm sea changed considerably in a relatively short time and in a restricted area. This resulted in a spectrum that is described as a substantial wind-generated wave system accompanied by the two newly recovered low-frequency lobes. The lobes, one under 20 and the other under $40^{\circ}$ angle with the main wave system, lead to a spectrum with a directional spreading as large as $120^{\circ}$ that covers all but $3 \%$ of the energy. The other characteristic quantities are an approximate significant wave height of $H_{\mathrm{S}}=12 \mathrm{~m}$ (measured at the Draupner platform), period $T_{\mathrm{Z}}=12.5 \mathrm{~s}$, and $k D=1.6$ at the local depth of $D=70 \mathrm{~m}$.

From the available 2D spectrum and the known physical parameters, linear random Draupner seas can be constructed analytically. To transform these linear seas into nonlinear seas, numerical simulations are needed with a nonlinear code, for which specific care is needed to take the wide directional spreading into account. 
A third-order nonlinear numerical code will be used that can deal with four-wave interaction and the Benjamin Feir instability. Beforehand, it can be argued that the effect of the Benjamin-Feir instability may be very mild, because the value of $k D=1.6$ of the sea is just above the critical value 1.36 , and the spreading is wide which could reduce the steepness as observed in extensive laboratory experiments of Latheef and Swan (2013). The steepness, which, for unidirectional waves, would give $k H s / 2=0.14$, turns out to be much larger, up to a factor 5 , in the simulations near high crests.

The code to be used is a pseudo-spectral implementation based on the Hamiltonian formulation of surface waves. The dynamics is governed by equations for the surface elevation and potential at the free surface only, without the necessity to calculate the interior flow. To get explicit approximate expressions in a consistent way, the kinetic energy functional is approximated explicitly in the surface variables. Using, in Dirichlet's principle, a generalization of classical Airy functions for which the constant depth is replaced by the total depth, the kinetic energy is found as a positive definite functional using Fourier integral operators. An approximation of this functional up to fifth order in nonlinear terms is taken and results in the third-order dynamics to be used here. The spectral implementation will deal with the correct dispersion properties for all frequencies in the broad spectrum.

Linear Draupner seas will be used as input for successive nonlinear simulations. The very broad directional spreading requires an influx scenario that assures that all directions are present in the domain of investigation. Instead of using a standard method to influx the waves from a straight line perpendicular to the main propagation direction, we designed a new influx scenario that also compensates the leaking of waves through open boundaries. The domain to be used is a semicircle of radius nearly $5 \mathrm{~km}$, and the boundary area is used as assimilation area where at discrete instances, the updated linear sea is smoothly merged with the results of the ongoing nonlinear simulation. In that way, an almost uniform nonlinear sea is obtained away from the boundary.

Quantitative and qualitative properties of the nonlinear waves are investigated in a smaller rectangular area of 15 $\mathrm{km}^{2}$ for an ensemble of 40 seas that were simulated over 200 wave periods each. Except for crest exceedance statistics from a limited number of buoys, we also calculate the elevation exceedance over the whole area. The simulations provide a database for statistical investigation which indicate that the Draupner wave height is not very exceptional. Studying some freak wave appearances in detail will show that it is mainly the constructive interference between oblique waves that lead to the extreme heights.

Section 2 starts with the description of the 2D Draupner spectrum, and the design of random linear Draupner seas. In Sect. 3, details of the numerical code are given followed by a detailed description of the influx scenario. In Sect. 4, results of the numerical investigations are reported, a mix of statistical information and qualitative description of extreme wave occurrences. The final section contains discussion and conclusions.

\section{Draupner spectrum and linear seas}

In the first subsection, the Draupner spectrum is described, after which the linear Draupner seas are constructed.

\subsection{Details of the Draupner spectrum}

New insights in meteorological circumstances mentioned above lead to a spectrum that is very wide. Figure 1 shows the 2D spectrum obtained from data of the European Centre for Medium-Range Weather Forecasts, Reading, UK, but rotated over an angle of $13^{\circ}$ in the western direction to have the mean energy flux directed from North to South. The spreading of the spectrum is restricted to $120^{\circ}$, neglecting $3.3 \%$ of the energy under larger angles. The energy at the sides is substantial: cutting the spectrum to a spreading of $60^{\circ}$ or $90^{\circ}$ reduces the energy content with 22.1 and $9.3 \%$, respectively. Integrating over all directions leads to a $1 \mathrm{D}$ point spectrum that can be well approximated as a JONSWAP spectrum, with a fourth power decay for high frequencies, see Fig. 1. The explicit approximation is given by

$S_{1}(\omega)=\int S_{2}(\omega, \theta) \mathrm{d} \theta \approx \alpha \omega^{-4} \exp \left(-\frac{5}{4}\left(\frac{\omega}{\omega_{\mathrm{p}}}\right)^{-4}\right) \gamma^{\nu}$

with $v=\exp \left(-\frac{1}{2}\left(\frac{\omega-\omega_{\mathrm{p}}}{\omega_{\mathrm{p}} \sigma}\right)^{2}\right)$. The parameters are given by $T_{\mathrm{p}}=14.45 \mathrm{~s}, \omega_{\mathrm{p}}=0.44 \mathrm{rad} / \mathrm{s}, \gamma=2.51$, and $\sigma=0.18$ for $\omega<\omega_{\mathrm{p}}$ and $\sigma=0.16$ for $\omega>\omega_{\mathrm{p}} ; \alpha$ has to be taken, such that the correct value of $H_{\mathrm{s}}=12 \mathrm{~m}$ for the nonlinear sea is obtained, which should be somewhat higher than $12 \mathrm{~m}$ for the linear sea as explained further on. It is to be noted that using prescribed standard values, $\sigma=0.07$ and 0.09 leads to optimal parameters with decay rate of power -5 , but a much less accurate approximation of the 1D Draupner spectrum, see also Korotkevich and Zakharov (2015).

\subsection{Analytic seas}

The bottom of the sea in the simulations is taken to be flat at a depth of $70 \mathrm{~m}$. The seas that will provide the influx data for seas to be simulated with the nonlinear code are linear; analytic seas are designed by taking a superposition of regular wave components each having a distinct frequency and propagation direction. For the actual construction, the 

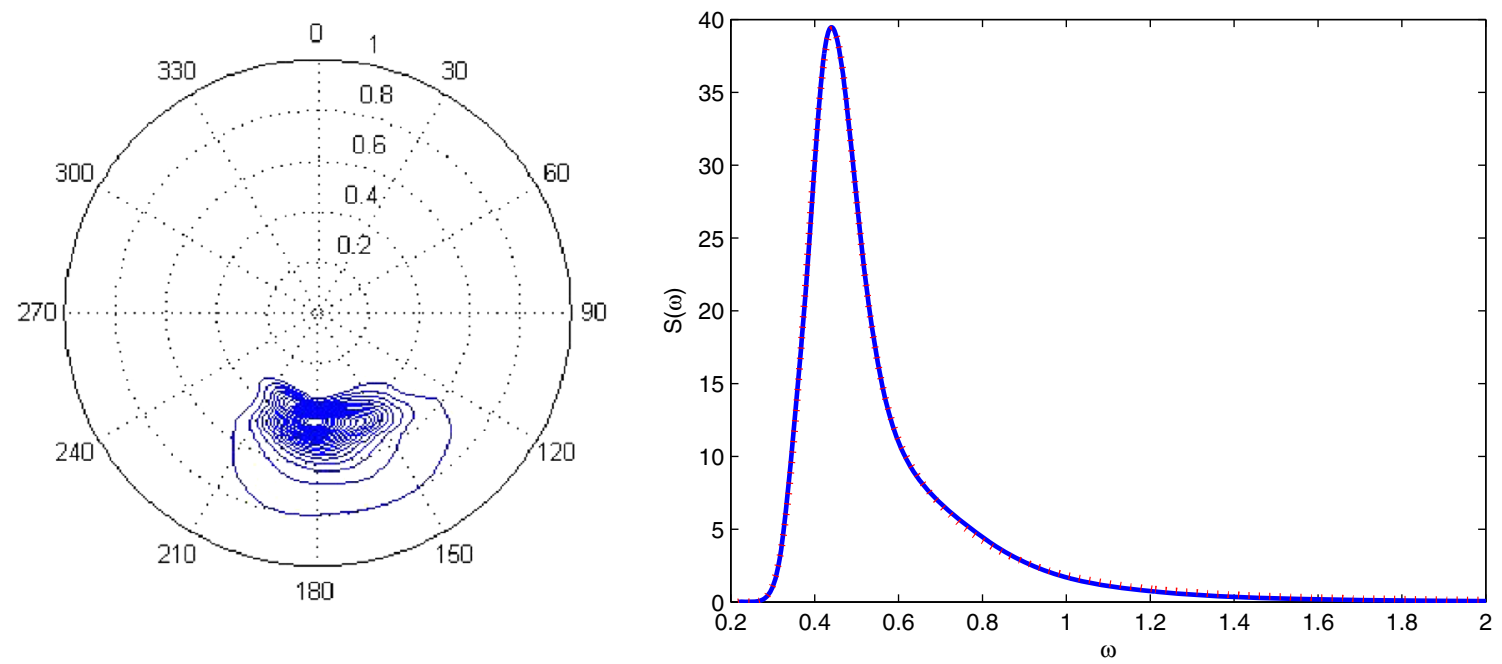

Fig. 1 At the left the 2D spectrum, rotated over an angle of $13^{\circ}$ to the west to have the main energy propagation to the South. At the right, the point spectrum (solid) with the approximated JONSWAP spectrum (dots)

wave spectrum $S(\omega, \theta)$ is discretised on an equally spaced set of frequencies $\omega_{m}$ covering the significant energy contributions. To assure that the sea is ergodic (Jefferys 1987), for each frequency, only a single direction is chosen by randomly drawing from the directional spreading which is used as a probability density function as in Goda (2010).

In more detail, writing $k=|\mathbf{k}|$ for the length of the wave vector $\mathbf{k}$, a linear sea has dispersion relation between frequency and wave vector given by $\Omega(\mathbf{k})=$ $\operatorname{sign}(\mathbf{e} \cdot \mathbf{k}) \Omega_{1}(k)$. Here, $\Omega_{1}(k)=\sqrt{g k \tanh (k D)}$ is the dispersion relation of unidirectional waves and $\mathbf{e}=(0,-1)$ the vector that defines the half space for propagating all waves towards the south. The analytic sea is then constructed as

$$
\begin{aligned}
\eta(x, y, t)= & \Sigma_{m} \sqrt{2 S\left(\omega_{m}\right) \mathrm{d} \omega} \cos \left(k_{m}\left(x \cos \theta_{m}+y \sin \theta_{m}\right)\right. \\
& \left.-\omega_{m} t+\alpha_{m}\right) .
\end{aligned}
$$

The summation is over frequencies $\omega_{m}$ that determine the wave numbers $k_{m}$ via $\Omega_{1}(k)$. The coefficients $\alpha_{m}$ are random phases in $[-\pi, \pi)$. The number of frequencies used to generate the influx signal is $36 * 20$. First, 36 equidistant frequencies $\omega_{M}$ are taken to cover the interval of relevant frequencies, with grid size $\Delta \omega$ say. At each of these 36 frequencies, the function $\theta \rightarrow S\left(\omega_{M}, \theta\right)$ is taken after normalization as probability distribution function from which the angles $\theta_{m}$ are obtained at 20 subfrequencies around and including $\omega_{M}$ in an interval of length $20 \mathrm{~d} \omega=\Delta \omega$.

For the influxing of waves in the numerical code, the corresponding surface potential is needed and will be obtained from the linear evolution equation $\partial_{t} \phi=-g \eta$.

\section{Numerical simulations}

In this section, we provide details of the numerical simulations. In the first subsection, the essentials of the HAWASSI$\mathrm{AB} 3 r d$-order code used for the numerical simulations are described; the acronym HAWASSI stands for Hamiltonian WAve Ship-Structure Interactions, and AB for Analytic Boussinesq, see the references for a link to the software. Then, the choice of the numerical domain is described and motivated by the wide directional spreading of the seas; details of the assimilation method are given that uses the linear analytic seas as boundary influx along a semicircle to get nonlinear effects in the interior. Some other numerical aspects are discussed in the last Sect. 3.3.

\subsection{Third-order HAWASSI-AB code for wave simulations}

The phase resolved simulations are executed with the HAWASSI-AB code that has been designed for the simulation of realistic waves in wave tanks, coastal and oceanic areas, and harbours. The underlying model is a set of Hamiltonian Boussinesq equations that has been discretised using a pseudo-spectral implementation, Kurnia and Van Groesen (2014, 2015, 2017). For linear waves, it has exact dispersion; nonlinear extensions in second-, third-, and higher order use Fourier integral operators to deal with the nonlinear phase speed operator that determines the kinetic energy expression. Simulations with the code have been tested for many cases against experiments and theory in simple and complicated geometries above flat and varying bottom, for harmonic and random waves, possibly breaking waves, and showed very 
good performance in relatively short simulation times. Yet, the code has not been tested against experimental data or other simulations of Draupner-like seas in 2D.

The basis of the model is the Hamiltonian formulation of surface waves, which is of Boussinesq type, i.e., it is dimension reduced using only the surface elevation $\eta(\mathbf{x}, t)$ and the potential at the surface $\phi(\mathbf{x}, t)$ as variables depending on horizontal directions $\mathbf{x}=(x, y)$. Zakharov (1968 for infinite depth) and Broer (1974 for flat bottom) discovered that the full irrotational surface wave equations can be written as a Hamilton system in $\eta, \phi$ as

$\partial_{t} \eta=\delta_{\phi} H(\phi, \eta), \partial_{t} \phi=-\delta_{\eta} H(\phi, \eta)$

where $\delta_{\phi} H$ and $\delta_{\eta} H$ denote the variational derivatives of the Hamiltonian $H(\phi, \eta)$ with respect to $\phi$ and $\eta$, respectively.

The Hamiltonian is, just as in Classical Mechanics, the sum of kinetic and potential energy

$H(\phi, \eta)=K(\phi, \eta)+P(\eta)$ with $\mathrm{P}(\eta)=\frac{1}{2} \int \mathrm{g} \eta^{2} \mathrm{~d} \mathbf{x}$.

The main challenge in this formulation is to approximate the kinetic energy in the surface variables explicitly. To achieve this, Dirichlet's principle is used, which expresses that for irrotational flows, the potential that minimizes the kinetic energy and has prescribed potential $\phi$ at the given surface elevation $\eta$ is obtained for the (unique) potential that describes incompressible flow, i.e., satisfies the Laplace equation. With Airy's theory, an exact expression for linear waves above constant bottom is possible and serves as a good starting point to get approximations for nonlinear and spatially inhomogeneous problems. In the Analytic Boussinesq (AB) model of HAWASSI software, Fourier expansions are used that lead to exact dispersion for linear waves. Using generalized Airy functions in which the constant depth $D$ is replaced by the total depth $D+\eta(\mathbf{x}, t)$, pseudo-differential operators are extended to Fourier integral operators. The kinetic energy functional can then be described as a quadratic functional in $\mathbf{u}=\nabla \phi$

$K(\phi, \eta)=\frac{1}{2 g} \int\left(|C(\eta) \mathbf{u}|^{2}+|B(\eta)(\nabla \eta \cdot \mathbf{u})|^{2}\right) \mathrm{d} \mathbf{x}$.

Here, $B(\eta)$ and $C(\eta)$ are symmetric operators depending on $\eta$ but not on its derivatives. Hence, the steepness enters the functional only if the contribution with $B(\eta)$ is taken into account. The linear case is obtained for $C=C_{0}$ and $B=0$, with $C_{0}$ the linear operator which has the linear phase velocity as symbol, leading to fully dispersive linear wave theory. Taking $B=0$ and a first-order expansion of $C(\eta)$ leads to the 2 nd order equations; only in third order, with $C$ expanded in second order and $B=B_{0}$ independent of $\eta$, the steepness comes most pronounced into the equations.
Explicit expansions for up to fifth-order equations are given in Kurnia and Van Groesen (2014); for the special case of flat bottom, the expansions lead to the same equations as in higher order spectral methods, first developed by West et al. (1987) and Dommermuth and Yue (1987), but the implementation in the $\mathrm{AB}$ code is directly in surface variables only.

In this paper, the third-order code will be used to support effects of four-wave interactions and effects on wave deformation and interactions, as shown in Adcock et al. (2015). $\mathrm{AB}$ has a wave-breaking mechanism with a kinematic initiation criterion based on the quotient of horizontal fluid velocity $U$ at the crest and the crest speed $C_{\text {crest }}$; after initiation, the breaking is modelled by the eddy-viscosity model of Kennedy et al. (2000). Using the rather low value for the kinematic breaking criterion of $U / C_{\text {crest }}=0.8$, none of our simulations breaking took place, even though for several waves, steep gradients of 0.6 and more were encountered. Only for the highest crest wave, breaking was noticed for a lower initiation value of $U / C_{\text {crest }}=0.7$.

\subsection{Numerical domain and influx scenario}

In this subsection, we explain the choice for the numerical domain and how we use the constructed linear analytic sea described in Sect. 2 as input for nonlinear simulations. Clearly, the aim is to have an observation area that is large enough to study peculiarities of the nonlinear sea, while practical restrictions on the size of the numerical domain have to be taken into account. For the numerical spectral implementation of the $\mathrm{AB}$ code, a rectangular domain is required.

The motivation for the influx scenario is suggested from the description of an initial value problem that starts with the initial data for the elevation and the potential from a linear sea. A successive nonlinear simulation will start to propagate the waves in all wave directions present in the linear sea, mainly southward. Oblique waves must be allowed to pass the lateral and southern boundaries of the numerical domain. On the other hand, without influx of waves from the northern and lateral boundaries, the part of the rectangular domain where each point gets information from all required directions, i.e. from directions in an upward pointing triangle with angle of $120^{\circ}$ (the domain of dependence of the point), becomes quickly smaller with increasing time, and after some time, all waves will have left the numerical domain. Based on these observations, for long time simulations, an influx scenario has to be used that provides wave influx through the boundaries into the domain, while the boundaries should be transparent for outgoing waves. Wave influx from a straight line is a common method, see Lie et al. (2014), but cannot be used efficiently at the East and West boundaries, because the main propagation direction is tangential there. Therefore, we designed an alternative influx scenario as follows. 
A large semicircle is taken in the rectangular domain. A merging area is constructed as the area in between the boundary of the semicircle and a vertical shift upwards of this boundary, the orange-coloured area illustrated in Fig. 2. The vertical shift is of the order of one-third of the peak wave length, shown somewhat larger in the figure for better visibility. Influx updates of the linear sea, consisting of elevation and surface potential in the outer, 'linear', area of the semicircle, are merged with the result of the nonlinear simulation at times of the update, using a smooth partition of unity of the data. Updates are given every one-third of the peak period. The merging essentially let the nonlinear terms grow from zero in the linear area to unity in the nonlinear area. This space dependency does not change the conservation of the Hamiltonian. Outflow of waves in the South in between updates will give some decrease of the Hamiltonian over the whole area, but will preserve the Hamiltonian over the nonlinear area in between updates, and also from one update to another.

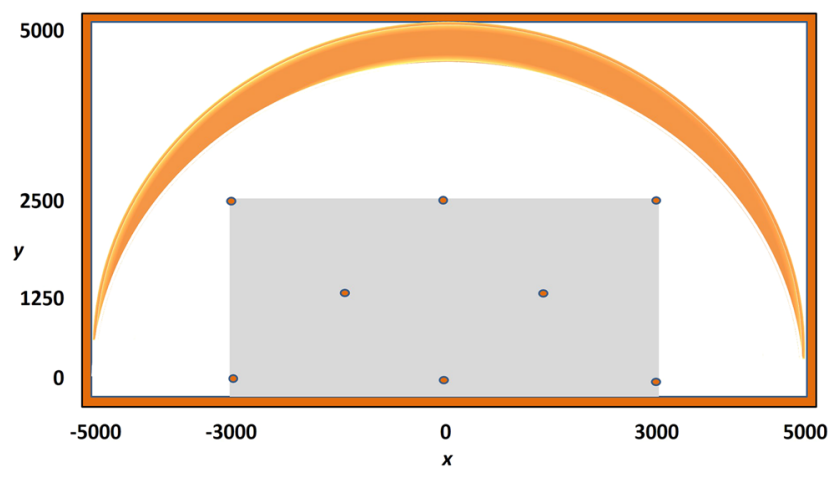

Fig. 2 Layout of the numerical domain with damping zones at the sides, the adjustment area for the influx scenario (shaded, orange), and the rectangular observation domain with the position of 8 buoys
The process of wave invasion from the outer region into the semicircle is illustrated in Fig. 3. Shown are the initial situation at $t=0$ with the linear sea in the outer region and vanishing elevation in the interior, and at successive times 5 , 10 , and 30 peak periods later, showing the gradual filling of the whole domain.

The rectangle is the domain $[-5200,5200] \times[-800$, 5200]; along all sides, there is a damping zone with width of $200 \mathrm{~m}$ at the north, east, and west sides, and of $500 \mathrm{~m}$ at the south. The semicircle has radius $4.75 \mathrm{~km}$.

Although the simulation is performed over the whole rectangle, we take a much smaller rectangle as observation domain where properties of the nonlinear sea will be investigated. This observation domain is the gray rectangle $[-3000,3000] \times[0,2500]$ in Fig. 2. Each point in this domain will receive contributions from all wave directions spread over $120^{\circ}$.

Eight buoys, also shown in the figure, are taken at positions B1 $(-3000,2500), \mathrm{B} 2(0,2500), \mathrm{B} 3(3000,2500), \mathrm{B} 4$ $(-1500,1250), \mathrm{B} 5(1500,1250), \mathrm{B} 6(-3000,0), \mathrm{B} 7(0,0)$, and B8 $(3000,0)$. The positions are such that each buoy is almost out of the dependence area of the other buoys, except buoys B6,7,8 which are below buoys B1,2,3 but $2500 \mathrm{~m}$ separated in the main propagation direction.

\subsection{Other numerical aspects}

For the case under consideration, bottom friction will be small and has, therefore, been neglected.

Each single sea is simulated for $3400 \mathrm{~s}$; subtracting 500 s to allow waves to enter the initially empty interior of the semicircle, the last $2900 \mathrm{~s}$ containing approximately 200 wave periods are used for analysing the sea.
Fig. 3 Illustration of the wave influx: left upper plot the initial situation with the linear sea in the North, right upper plot after 5 periods when nonlinear waves started to develop southwards, and in the lower plots, the further development after 10 and 35 wave periods of time

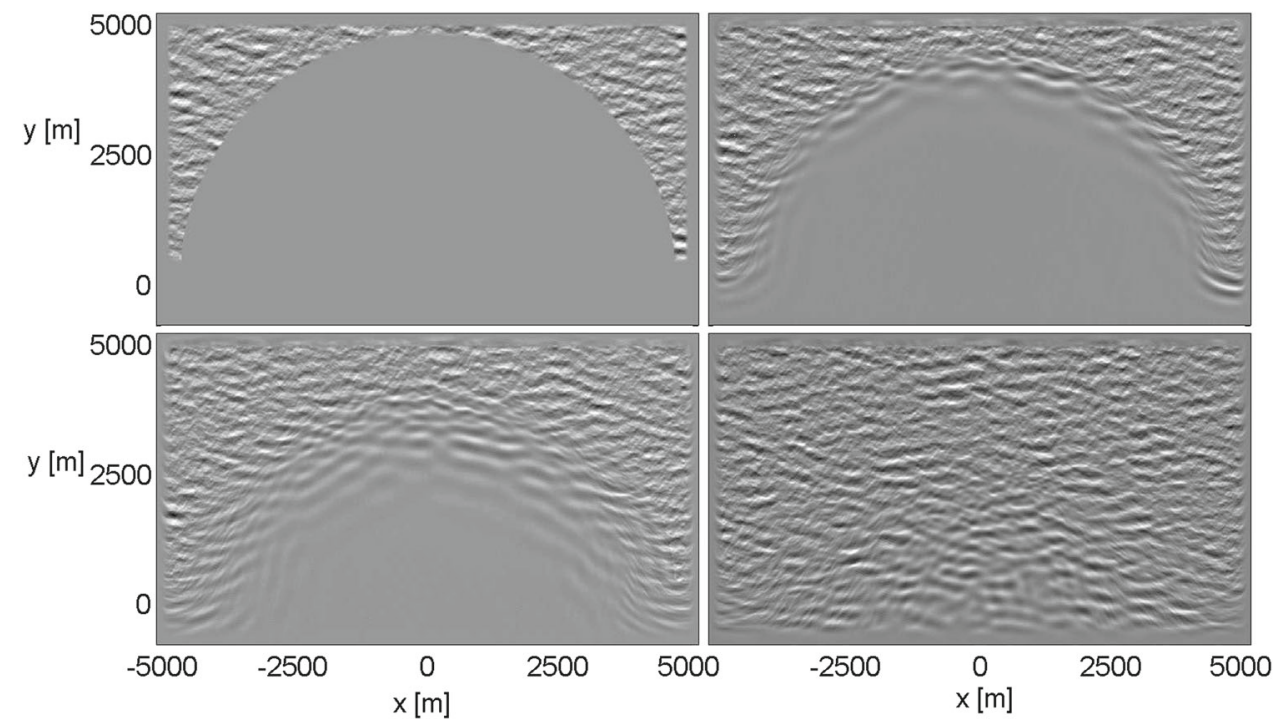


Table 1 Sensitivity for time-space discretization and nonlinearity from averaged data at 8 buoys

\begin{tabular}{lllllllll}
\hline Data source & $H_{\mathrm{s}}$ & $T_{\mathrm{p}}$ & $T_{m 01}$ & MTC & MTT & Skew & Asymm & Kurt \\
\hline sea $17 \mathrm{~m} 13$ & 11.90 & 13.68 & 11.53 & 12.39 & -10.15 & 0.181 & 0.0034 & 3.021 \\
$17 \mathrm{~m} 13 \mathrm{~d} t=1$ & 11.90 & 13.68 & 11.53 & 12.32 & -10.22 & 0.181 & 0.0343 & 3.022 \\
$17 \mathrm{~m} 13$ coarse & 11.56 & 13.68 & 12.30 & 11.68 & -9.68 & 0.126 & 0.0039 & 3.032 \\
$17 \mathrm{~m} 13$ linear & 12.24 & 14.76 & 11.44 & 12.62 & -11.10 & 0.180 & -0.0097 & 2.968 \\
\hline
\end{tabular}

Fig. 4 At the left, the plot of the point spectrum obtained from data of the 8 buoys in the ensemble (red dotted) compared to the design point spectrum from the 2D Draupner spectrum (blue solid). At the right, the plot of the significant wave height calculated at each point for the 8000 waves in the ensemble

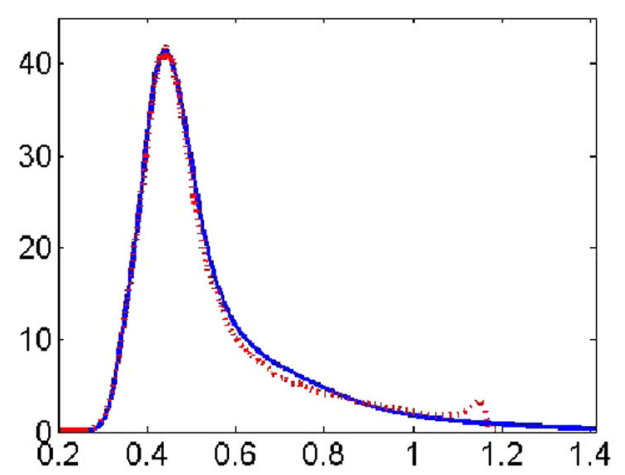

The grid size used for the simulations and for the postprocessing is $\mathrm{d} x=10.17 \mathrm{~m}, \mathrm{~d} y=11.74 \mathrm{~m}$, and for the time step, $\mathrm{d} t=0.5 \mathrm{~s}$. Investigation of robustness and sensitivity is quantified in Table 1 using data from the 8 buoys. In this and in the following, we use abbreviations as follows: $T_{m 01}$ is the inverse of the mean frequency of the spectrum, In this table, MTC stands for the Maximal Temporal Crest height, MTT for the Minimal Temporal Trough depth, Skew is the Skewness, Asymm the Asymmetry, and Kurt the Kurtosis. The simulation results show no relevant differences when the time step is doubled. Making the grid size coarser by a factor 2 in each direction does lead to a noticeable smaller value for $T_{m 01}$. This may be explained from the fact that the frequency cutoff for this coarser grid is at 0.82, compared to 1.2 for the finer grid, see Fig. 4. The different value of $H_{\mathrm{s}}$ for the linear sea is almost as prescribed, as will now be explained.

In the dynamic influx assimilation method, we use a linear sea that has to be taken, so that the required spreading and the desired significant wave height are obtained for the nonlinear sea. One effect of nonlinearity that was observed is a difference between the significant wave height of a linear sea and the significant wave height of the nonlinear sea that is simulated using influx data from the same linear sea. Such a difference can be explained as follows. For linear waves, the equipartition of energy holds: averaged over time, the potential energy is equal to the kinetic energy. However, for nonlinear waves, the kinetic energy is larger than for linear waves. Since the Hamiltonian, which is the sum of kinetic and potential energy is exactly conserved in our Hamiltonian formulation, in a confined domain without netto in- or outflow, in the transition from linear to nonlinear waves, the poten-
Table 2 Sensitivity in simulating the highest crest

\begin{tabular}{llllrl}
\hline Data source & mean $H_{\mathrm{s}}$ & Crest height & \multicolumn{1}{l}{$X$} & \multicolumn{1}{l}{ Time } \\
\hline sea $17 \mathrm{~m} 13$ & 12.02 & 21.7 & -900 & 785.1 & 2497 \\
$17 \mathrm{~m} 13 \mathrm{~d} t=1$ & 12.02 & 21.7 & -900 & 785.1 & 2498 \\
$17 \mathrm{~m} 13$ coarse & 11.71 & 15.3 & -967 & 1317.7 & 2460 \\
$17 \mathrm{~m} 13$ linear & 12.37 & 16.4 & -920 & 937.8 & 2508 \\
\hline
\end{tabular}

tial energy must become less. Since the potential energy is related to the variance of the surface elevation, the nonlinear sea will have lower significant wave height than the linear sea used for influxing. We observed this difference between kinetic and potential energy for all seas, and actually also for simulations of other cases. At this moment, we cannot yet quantify this difference theoretically a priori, but with a linear influx that is $2.5 \%$ higher, the nonlinear seas have approximately the targeted value $H_{\mathrm{s}}=12 \mathrm{~m}$, as is shown in the plot at the right in Fig. 4.

In Table 2, we show the sensitivity in finding the highest crest over the whole observation area. This shows more severe differences for the coarse grid: the most extreme height in the coarse simulation is almost one-third lower than for the fine grid. Moreover, the highest crest appears some $40 \mathrm{~s}$ earlier, leading to a difference of position in the propagation direction of $530 \mathrm{~m}$, which is quite consistent for propagation with the group velocity. In the lateral direction, there is a smaller difference of $67 \mathrm{~m}$. Note that the data for the linear simulation are even better than for the nonlinear simulation with the coarse grid. 


\section{Quantitative and qualitative aspects of Draupner seas}

Properties of 40 seas were investigated in the rectangular observation domain with a total of more than 8000 waves passing each point. Quantitative and statistical results are reported in the first subsection, for the ensemble but also for two seas, ensemble numbers $17 \mathrm{~m} 13$ and $17 \mathrm{~m} 26$, that are considered in the next subsections.

All the seas in the ensemble show complex patterns and dynamics leading to a variety of phenomena that cannot easily be described in a comprehensive way. Events like extreme crests can be observed and studied locally, but the complicated interactions that lead to such events seem to require a global spatial view coupled to dynamical interactions. Although nonlinearity deforms the wave patterns somewhat, it seems quite well possible to explain the local processes as superposition of waves of different amplitude, wave length (speed), and directionality, so that at a specific position and time coherent or destructive interference of the various waves determines the elevation. Nonlinearity will mainly influence the wave heights and local speeds of the waves.

The spatial and temporal processes will be described in the next two subsections for two seas that contain the highest waves encountered in the whole ensemble: a succession of three freak crest heights found in $17 \mathrm{~m} 13$ and a single freak wave in $17 \mathrm{~m} 26$.

\subsection{Quantitative results}

The point spectrum calculated from data at the 8 buoys of all ensemble seas is compared to the design point spectrum from the 2D Draupner spectrum in Fig. 4. This shows that the target 1D spectrum is well recovered, but due to the restricted mesh size, a cutoff at $\omega=1.2 \mathrm{rad} / \mathrm{s}$ appears. The directional spectrum has been calculated at several points and agreed well with the target spectrum.

The significant wave height, calculated at each point in the domain over 8000 waves, is shown in Fig. 4 also. The averaged value over the ensemble and the domain is $H_{\mathrm{s}}=$ $12.04 \mathrm{~m}$, with variations within 11.8 and $12.4 \mathrm{~m}$ outside a small area near the mid south of the domain.

An overview of the most extreme crest and trough values that were encountered in the ensemble is given in Fig. 5, where for each of the 40 seas, the maximal and minimal elevation are plotted together with the significant wave height of the sea. The deviation in $H_{\mathrm{s}}$ of the 40 seas from the averaged value $H_{\mathrm{s}}=12.04 \mathrm{~m}$ is at most $0.1 \mathrm{~m}$.

A well-known effect of nonlinearity is illustrated in Fig. 6 that shows the Maximal and Minimal Temporal Area Amplitude, i.e., the maximal and the minimal elevation at each time (horizontally) over the whole area, for a linear and nonlinear simulation of the same input (sea $17 \mathrm{~m} 13$ in the ensemble).

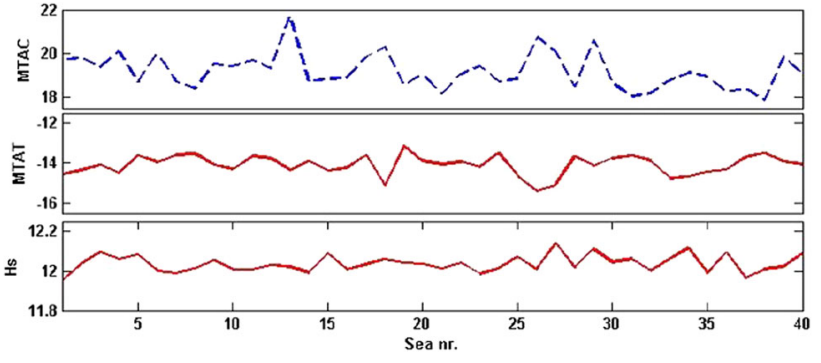

Fig. 5 For each of the 40 seas horizontally, the upper and middle plot show the maximal temporal area crest height (MTAC) and the minimal temporal area trough depth (MTAT) and the lower plot the significant wave height $H_{\mathrm{s}}$

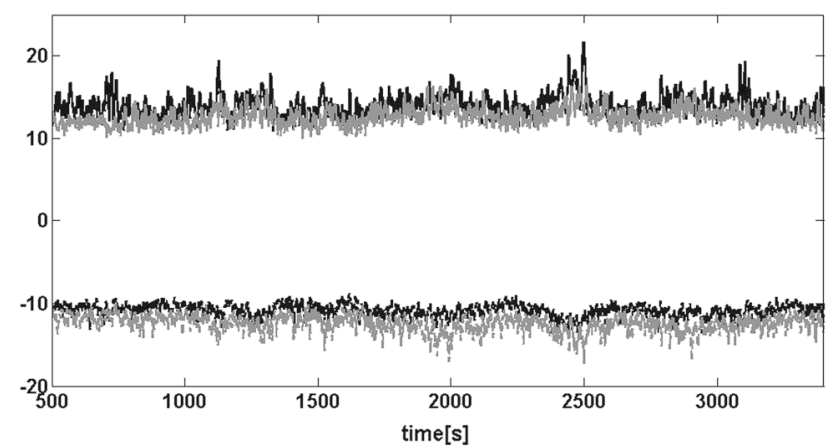

Fig. 6 Plot of the maximal and minimal temporal area amplitude for sea $17 \mathrm{~m} 13$. Data for the linear sea are in gray and for the nonlinear sea in black

As expected, higher crests and higher troughs are found for the nonlinear sea, despite the fact that the significant wave height is higher for linear seas than for the corresponding nonlinear sea as discussed in Sect. 3.3.

Data from the buoys are assembled in Table 3. Data in the first row are for the ensemble, and below that the data are for nonlinear and linear seas $17 \mathrm{~m} 13$ and $17 \mathrm{~m} 26$.

The statistical crest and trough exceedance measured from data at the 8 buoys over the total ensemble are shown in Fig. 7 at the left. To improve these buoy exceedance results, the elevation exceedance at all grid points in the domain over the whole ensemble has been calculated. This is motivated by Cavaleri et al. (2016) in which the notion of encounter probability was mentioned that should serve as an additional indicator of extreme waves.

The elevation exceedance probability $p_{\text {area }}(\alpha)$ is the fraction of the area at which the elevation exceeds $\alpha H_{\mathrm{s}}$ averaged over time. Note that for $\alpha=0$, the fraction of positive area can be found, which turns out to be $48 \%$ of the area, lower than $50 \%$ that can be expected for linear seas. From the elevation exceedance as cumulative probability, the probability density is obtained by differentiation, from which crest and through exceedance can be found. The elevation exceedance and the density are given in Fig. 8; the crest probabilities are 
Table 3 Averaged data from the 8 buoys for the total ensemble and for two seas simulated linearly and nonlinearly

\begin{tabular}{lllllllll}
\hline Data source & $H_{\mathrm{s}}$ & $T_{\mathrm{p}}$ & $T_{m 01}$ & MTC & MTT & Skew & Asymm & Kurt \\
\hline Ensemble & 12.01 & 14.23 & 11.49 & 13.76 & -10.71 & 0.190 & 0.0036 & 3.056 \\
\hline Sea 17m13 & 11.90 & 13.68 & 11.53 & 12.39 & -10.15 & 0.181 & 0.0340 & 3.021 \\
$17 \mathrm{~m} 13$ linear & 12.24 & 14.76 & 11.44 & 12.62 & -11.10 & 0.180 & -0.0097 & 2.968 \\
\hline Sea 17m26 & 11.95 & 14.89 & 11.61 & 15.90 & -11.14 & 0.203 & -0.0054 & 3.087 \\
$17 \mathrm{~m} 26$ linear & 12.31 & 15.05 & 11.49 & 13.42 & -12.99 & 0.173 & 0.0189 & 2.990 \\
\hline
\end{tabular}
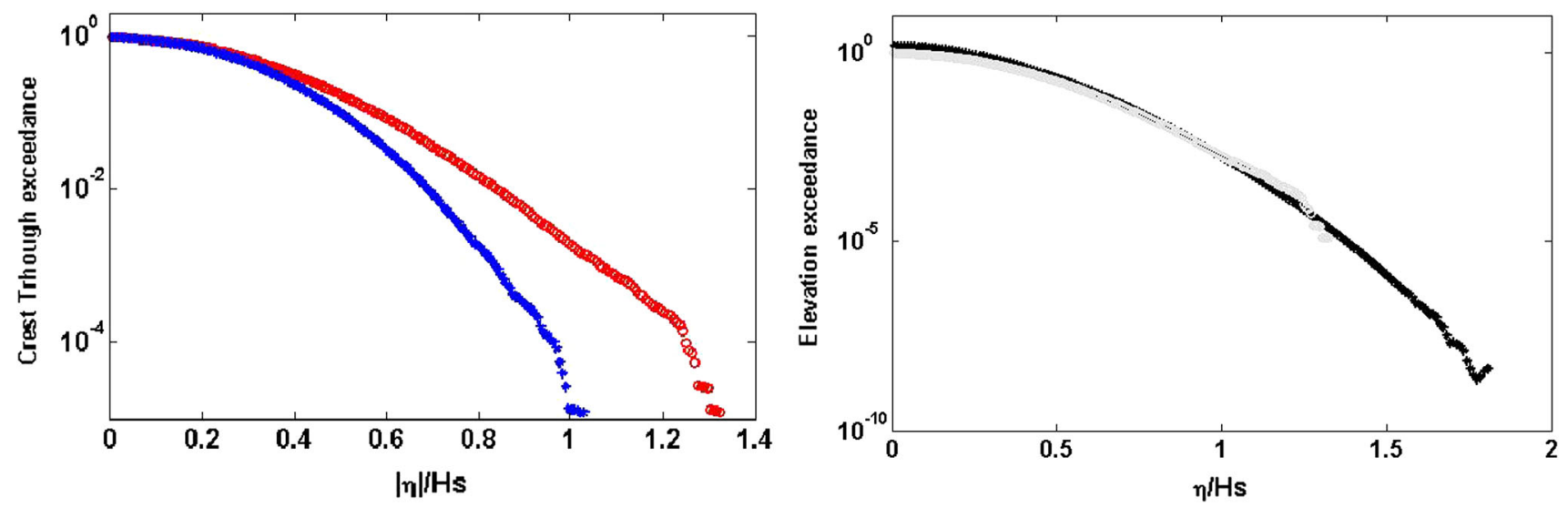

Fig. 7 At the left, the plot of the crest exceedance (black) and trough exceedance (gray) calculated from 8 buoys for 8000 waves. At the right, the crest exceedance obtained from the elevation exceedance (black) with the crest exceedance from 8 buoy data (gray) for comparison

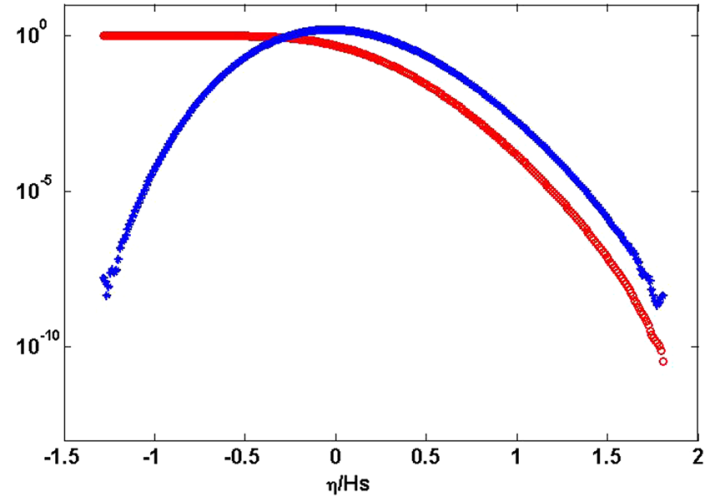

Fig. 8 Plot of the elevation exceedance: cumulative distribution (red) and probability distribution (blue) obtained from all ensemble data

shown again in the right plot of Fig. 7 to compare the results obtained from the 8 buoys and from all grid points.

In Table 4, explicit values are provided for the crest exceedance on the interval where inaccurate values from differentiation of the area exceedance are excluded; for comparison, also the values from buoy observations are given.

\subsection{Three successive freak crests}

Three successive crest heights larger than $1.5 H_{\mathrm{S}}$ are present in sea $17 \mathrm{~m} 13$ and will be described in this subsection. These
Table 4 Probabilities of crest exceedance from 8 buoys (second column) and as calculated from the elevation exceedance (third column)

\begin{tabular}{lll}
\hline Exc. value $\eta / H_{\mathrm{S}}$ & $\begin{array}{l}\text { Crest Exc. from } \\
8 \text { buoys }\end{array}$ & $\begin{array}{l}\text { Crest Exc. from } \\
\text { elevation Exc. }\end{array}$ \\
\hline 1.0 & $1.8 \mathrm{e}-3$ & $1.3 \mathrm{e}-3$ \\
1.1 & $7.1 \mathrm{e}-4$ & $5.0 \mathrm{e}-4$ \\
1.2 & $2.4 \mathrm{e}-4$ & $1.5 \mathrm{e}-4$ \\
1.3 & $2.2 \mathrm{e}-5$ & $3.4 \mathrm{e}-5$ \\
1.4 & $\mathrm{x}$ & $6.8 \mathrm{e}-6$ \\
1.5 & $\mathrm{x}$ & $1.4 \mathrm{e}-6$ \\
1.6 & $\mathrm{x}$ & $2.2 \mathrm{e}-7$ \\
\hline
\end{tabular}

crest heights satisfy the criterion of a freak wave, but do not fully satisfy the requirement that they come 'unexpectedly', since one follows after the other. In fact, the crests are from two waves, with the first wave travelling over a large distance during almost 2 periods with large crest height, during which two times the height exceeds $1.5 H_{\mathrm{s}}$; after this, the successive wave achieves the largest height of $1.8 H_{\mathrm{s}}$. Since the crests appear at three different positions, and only two waves are involved, this seems somewhat different than a 'three sisters' phenomenon (Seyffert et al. 2016). This whole freak appearance takes place in the time interval [2432,2508] of $76 \mathrm{~s}$ during which wave heights above $12 \mathrm{~m}$ are only found in the area $[-1300,-500] \times[0,2500]$. 
Fig. 9 Elevation plot at the position of highest crest as function of time over $1000 \mathrm{~s}$ and in the lower plot a zoom in near the time of the freak event
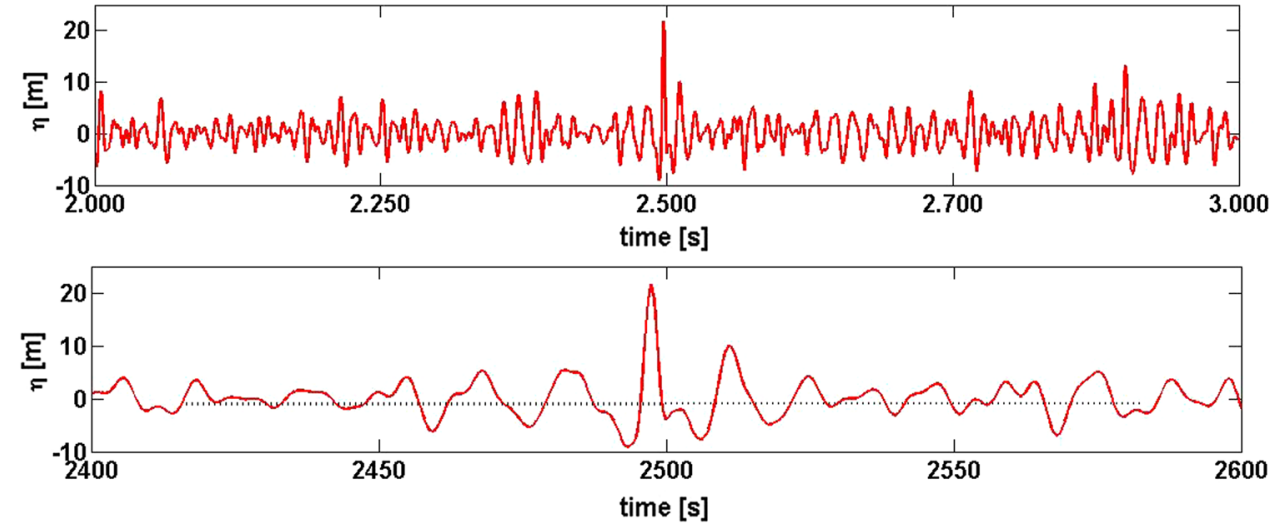

For this specific sea, data at the buoy positions were listed in Table 3. In addition, over the whole area, the peak period is $13.68 \mathrm{~s}$, the significant wave height is $H_{\mathrm{s}}=12.02 \mathrm{~m}$, the maximal elevation $21.7 \mathrm{~m}$, and the minimal trough depth $-14.4 \mathrm{~m}$. The wave length at peak frequency is $\lambda_{\mathrm{p}}=270 \mathrm{~m}$ and phase and group velocity values are $C_{\mathrm{p}}=19.8 \mathrm{~m} / \mathrm{s}$ and $V_{\mathrm{p}}=12.4 \mathrm{~m} / \mathrm{s}$.

The highest wave in this sea, and the highest one encountered in the whole ensemble, appears at $X=-900, Y=$ $785, t_{\text {event }}=2497.5$ and has crest height $21.73 \mathrm{~m} \approx 1.8 H_{\mathrm{s}}$. The time trace at the extreme crest is given in Fig. 9. The single high peak in this plot resembles the peak in the time trace of the original Draupner wave. The wave height is approximately $30 \mathrm{~m} \approx 2.5 H_{\mathrm{s}}$.

The downstream propagation along a line in the $y$ direction, which is the main propagation direction, in Fig. 10 shows three different snapshots in two plots. In these plots, the Maximal and Minimal Temporal Amplitudes (MTA's, black and cyan dashed, respectively) are also shown: the maximal, resp. minimal, elevation during a time interval of $200 \mathrm{~s}$, approximately 14 periods centred around $t_{\text {event }}$. In the upper plot, a very high wave (solid blue) with crest height $19.75 \mathrm{~m}$ $\approx 1.65 H_{\mathrm{s}}$ is observed at time $t=2442.5 \approx t_{\mathrm{event}}-4 T_{\mathrm{p}}$ near $y=1666 \mathrm{~m}$. This wave propagates downstream (to lower $y$-values) during which it retains a high value with another peak value of $18.93 \mathrm{~m}$ at time $t=2466 \approx t_{\text {event }}-2.3 T_{\mathrm{p}}$ at position $y=1161$ (blue dashed). In that time, it traveled a distance of $505 \mathrm{~m}$. This crest defines the part of the Maximal Temporal Amplitude in between the high points. This wave, plotted again in the lower plot, then decreases in amplitude and transfers its energy to its succeeding wave (red solid) which after $2.3 T_{\mathrm{p}}$ reaches the highest crest value at $Y$, a distance $375 \mathrm{~m}$ further downstream. Note that because this is a plot on one vertical line, in a neighbourhood of the first two high waves on this transect, there may be points with slightly higher elevation.

The downstream propagation along the same vertical transect is now investigated by looking at the dynamics in the main propagation direction along the whole transect trough
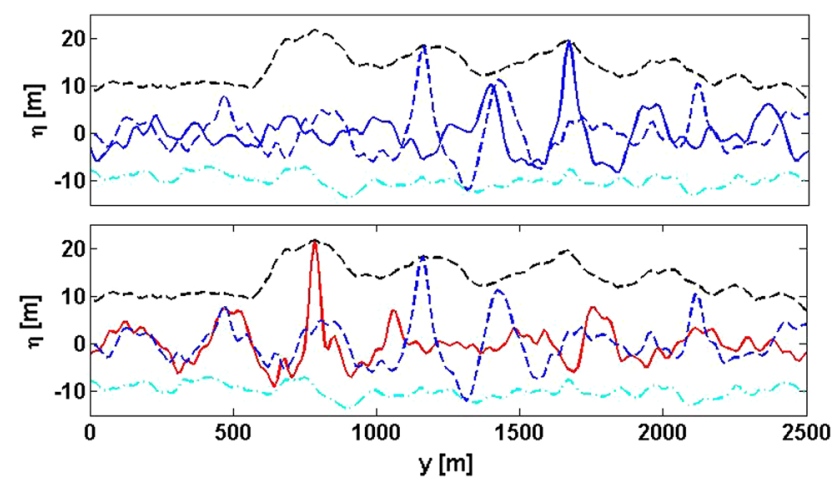

Fig. 10 Plot of the elevation as function of $y$ at three successive times showing the three high crests. In the upper plot, the first wave (solid blue) and its evolution to the second extreme height (blue dashed). In the lower plot, the same (blue dashed) second crest and the highest third crest on the successive wave (solid red)

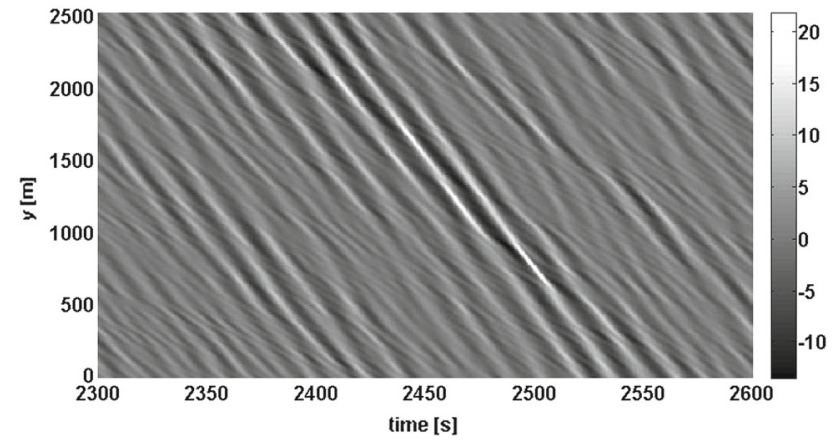

Fig. 11 Plot of elevation at $x=X$ as function of time (horizontal) in the main propagation direction $y$ (up to down)

$x=X$. The result is shown in Fig. 11 as plot of the elevation as function of $y$ and $t$. The slope of the crest trajectories is the component of the velocity in the $y$-direction at that position and time. Near the three high crests described above, it can be observed that the waves that contribute to the elevation at these events travel with the same $y$-component of the velocity; and from the steepness of the trajectories, it can be concluded that the speed is larger compared to the speed at 
other positions and times. This large speed is actually slightly larger than the phase speed $C_{\mathrm{p}}$ at peak frequency of the most energy carrying waves, which implies that the wave direction must be almost southward, clearly visible in dynamic simulations of the waves.

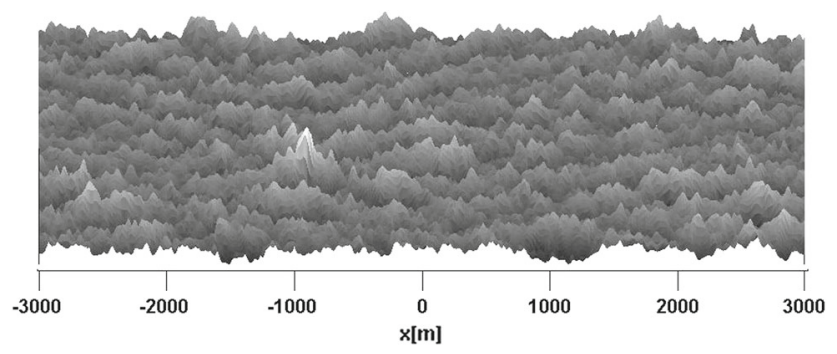

Fig. 12 Plot of the nonlinear sea state at time $t=2497.5$ looking from south (bottom) to north over the whole observation area. The highest crest, with white cap, is at the intersection of two oblique crests, at $x=-900, y=785$
A broader spatial-temporal view of the sea near the appearance of the three successive freak crests is now given using several snapshots in different ways of presentation. A snapshot of the sea over the whole observation area is given in Fig. 12 at the time $t=2497.5$ that the highest crest occurs at $x=-900, y=785$. The plot shows, apart from the highest crest, a typical view of a somewhat confused sea. Crest lines of more than $1 \mathrm{~km}$ length are directed somewhat in south-eastern and south-western direction, causing a pattern that is much shorter in the propagation direction than in the transversal direction. The highest elevation is at the intersection of two oblique crests, seemingly causing constructive interference that contributes to the isolated high crest.

The dynamic evolution is difficult to capture in snapshots; in the entire surrounding of the successive freak wave area active interaction is visible between waves, resulting in locally different downward propagation speeds, deforming crests, and changing heights along crests. To illustrate the dynamics resulting in the three high crests, in Fig. 13, in
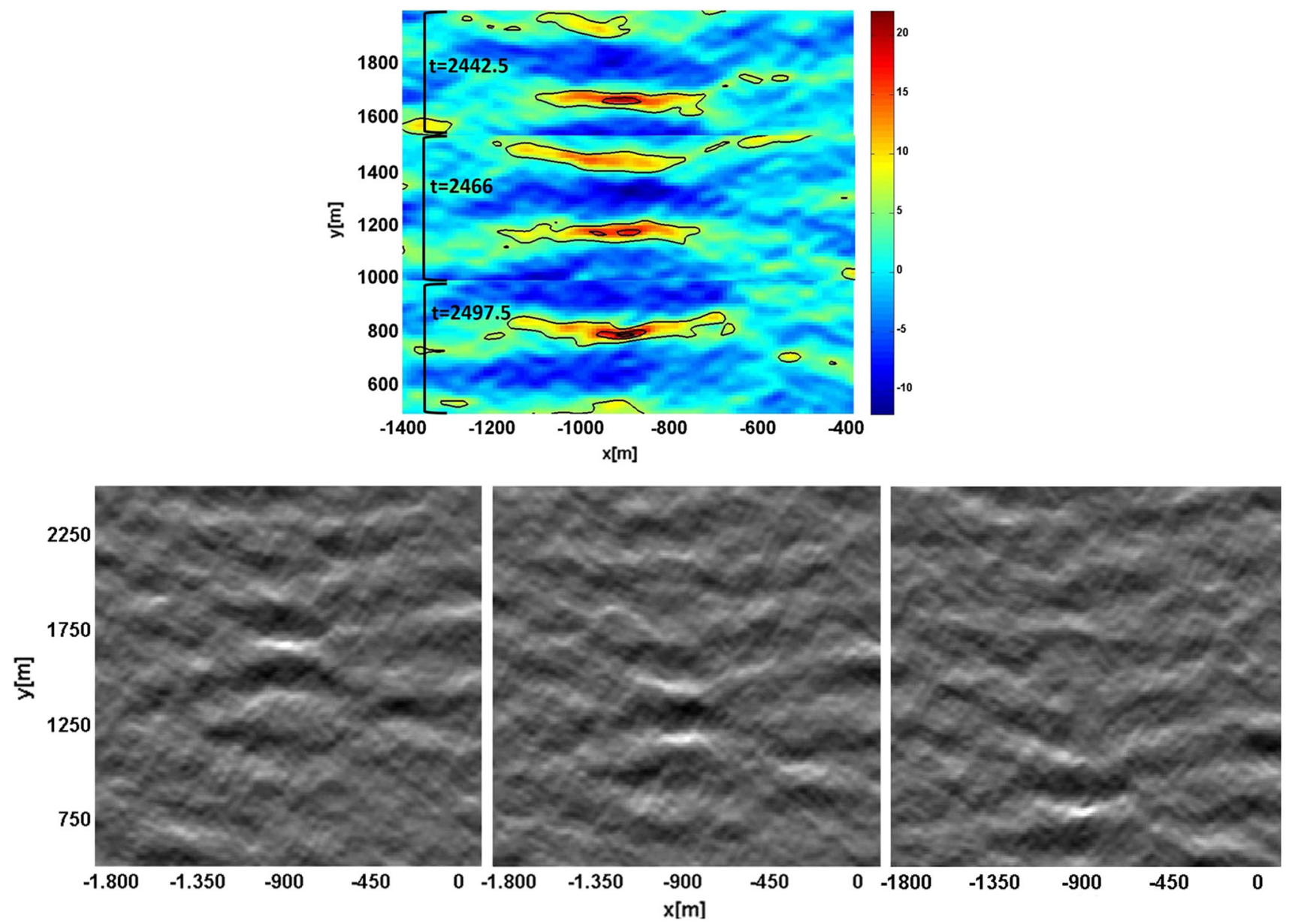

Fig. 13 In the upper plot, the three successive freak crests are shown in one area but at different times: in the northern part at $t=2442.5 \mathrm{~s}$ the wave of height $19.57 \mathrm{~m}$, in the middle part the crest height 18.22 $\mathrm{m}$ at time $t=2466 \mathrm{~s}$, and in the southern part the highest freak wave at $t=2497.5 \mathrm{~s}$ with crest height $21.7 \mathrm{~m}$. Level lines are shown for values 6,16 and $20 \mathrm{~m}$. In the lower plots, the three successive freak crests are shown at the three different times in a somewhat larger area 
Fig. 14 Plot of the minimal trough depth (lower than $9 \mathrm{~m}$ ) at the left and of the maximal crest height (higher than $9 \mathrm{~m}$ ) at the right over a time interval of $120 \mathrm{~s}$ for the three successive freak crests
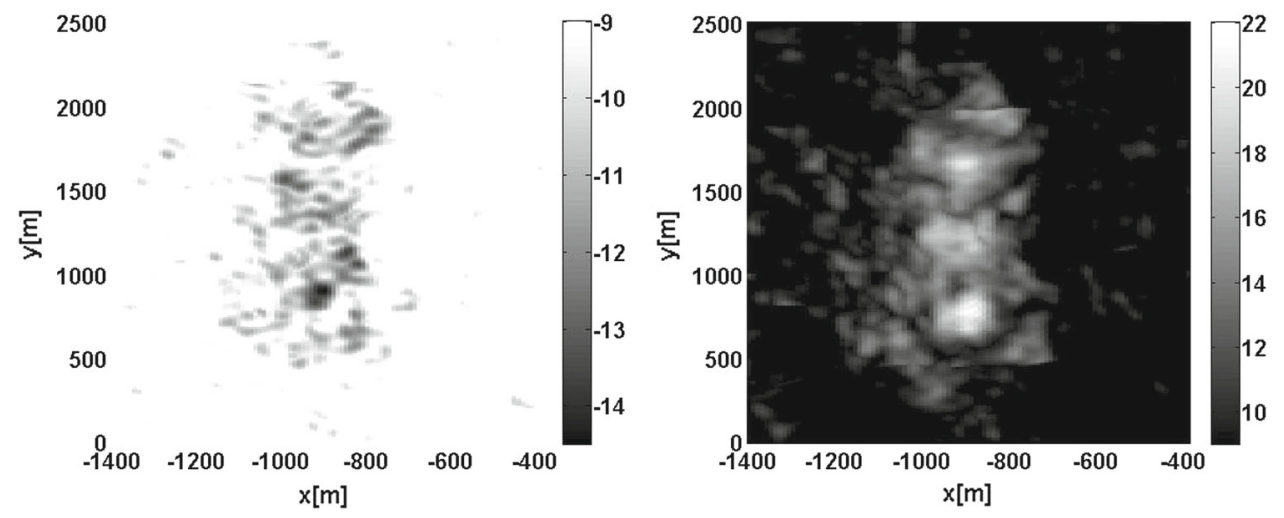

one area $[-1400,-400] \times[500,2000]$, we show three snapshots of the waves at different times, giving an indication of the southward propagation. The first freak wave of height $19.57 \mathrm{~m}$ is shown in the northern part of the plot at time $t=2442.5 \mathrm{~s}$. The crest propagates quickly southward and reaches with another peak value of $18.22 \mathrm{~m}$ at time $t=2466$ $\mathrm{s}$ the position shown in the middle part of the plot. The lower part of the plot is at $t=2497.5 \mathrm{~s}$ and contains the highest freak wave with height $21.7 \mathrm{~m}$. At each of the three times of the extreme crests, Fig. 13 also shows plots of the sea in a somewhat larger area.

To illustrate the localized spatial extend of the three successive freak crest appearance, in Fig. 14, the maximal and minimal elevations are shown in a time interval of $120 \mathrm{~s}$ starting $30 \mathrm{~s}$ before the first high crest. Only elevations of more than $9 \mathrm{~m}$ are shown which implies that such large elevations are not present in the lateral direction outside the area of the freak event.

\subsection{Single freak wave}

A single freak wave was observed in sea $17 \mathrm{~m} 26$ and will be described in this subsection. Data at the buoy positions were listed in Table 3. Over the whole area, the peak period is $T_{\mathrm{p}}=14.89 \mathrm{~s}$, the significant wave height is $H_{\mathrm{s}}=12.01 \mathrm{~m}$, the maximal elevation $20.7 \mathrm{~m}$, and the minimal trough depth $-15.4 \mathrm{~m}$. The wave length at peak frequency is $\lambda_{\mathrm{p}}=308 \mathrm{~m}$ and phase and group velocity values are $C_{\mathrm{p}}=20.7 \mathrm{~m} / \mathrm{s}$ and $V_{\mathrm{p}}=13.8 \mathrm{~m} / \mathrm{s}$. The highest wave occurred at $t=3115, x=$ $-1286, y=1948$. The data and further investigations show that it is an impressive, but fully isolated occurrence caused by interactions of waves from different directions. In Fig. 15, time traces of the elevation at the position of the highest crest are shown for all observation time and a zoom in for $350 \mathrm{~s}$ around the highest crest. Note that early in the full time trace, there was another freak wave at the same position.

Figure 16 shows at the left the surrounding area of the highest crest at the time of the freak event; level lines of 12 and $18 \mathrm{~m}$ surround the highest crest. The plot at the right

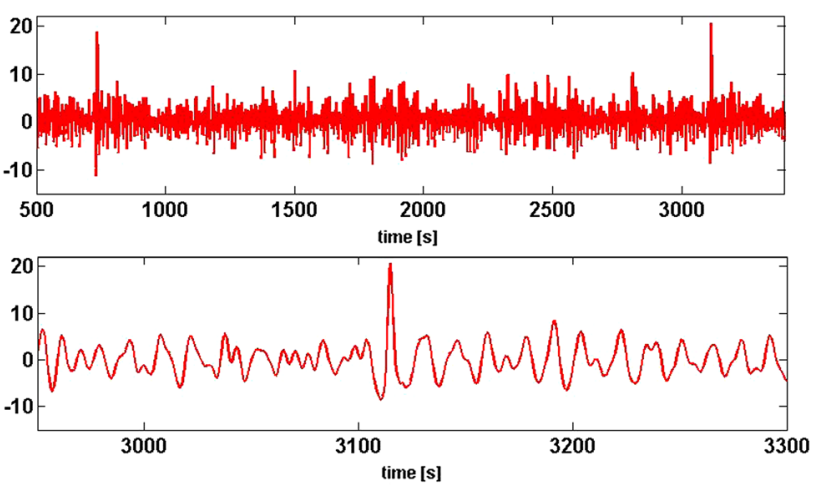

Fig. 15 For the single freak wave, shown are the time trace of the elevation trough the highest crest on the full observation time length, and a zoom in around the maximal height for $350 \mathrm{~s}$

shows at the same instant the steepness (norm of the gradient of the surface elevation) in a somewhat smaller area; values of the gradient for the highest crest run from -0.73 to 0.70 , so also very steep at the back of the wave.

Plots of the maximal and minimal elevation in a time interval of length $120 \mathrm{~s}$ around the time of the event in Fig. 17 show the localized extent of this extreme wave in a very moderate surrounding. Figure 18 shows the elevation at the time of the freak event at the vertical transect through the event position, and the lower plot gives the space dynamics at the same transect for all $y$-values, showing that the freak wave produces highest speeds in the main propagation direction.

\section{Discussion and conclusions}

The somewhat technical adjustment to rotate the original spectrum, so that the main energy flux is from North to South, has helped considerably to design the most optimal influx domain and to interpret the wave evolution in the numerical output. The semicircle with assimilation from the outer area can deal successfully with the wide spreading of the sea, so that each point in the observation area receives waves from all relevant directions. The plot of the significant wave height 

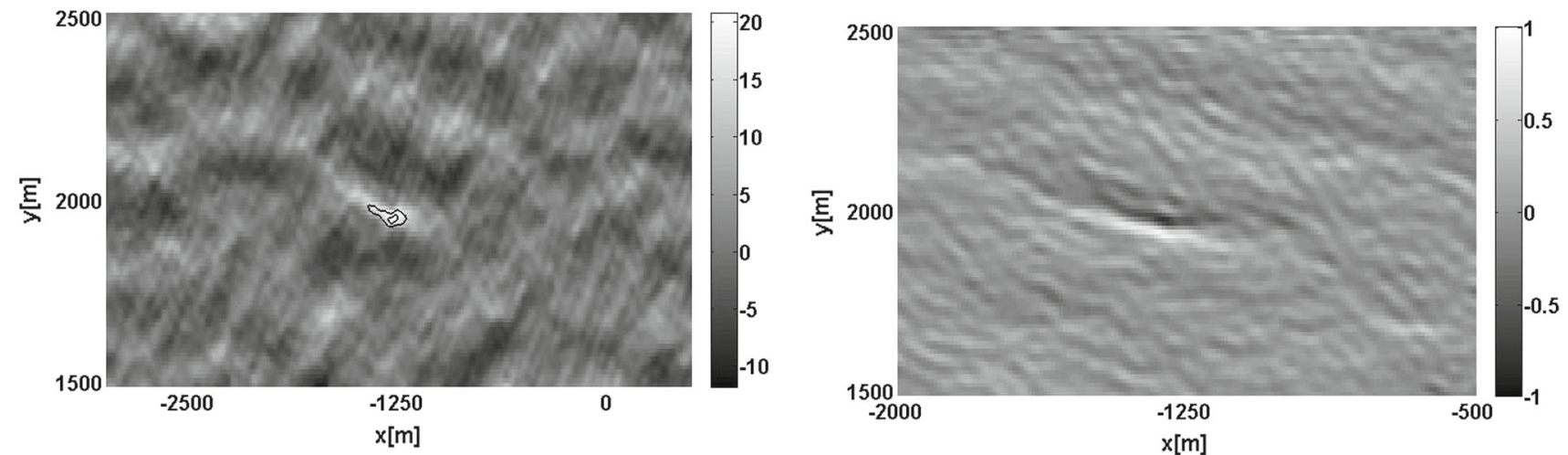

Fig. 16 At the left, the elevation in an area around the single freak wave, and at the right, the steepness in a smaller domain

Fig. 17 Plots of the minimal (left, lower than $-9 \mathrm{~m}$ ) and maximal (right, higher than 9 m) elevations over a time interval of $120 \mathrm{~s}$ around the time of the highest crest

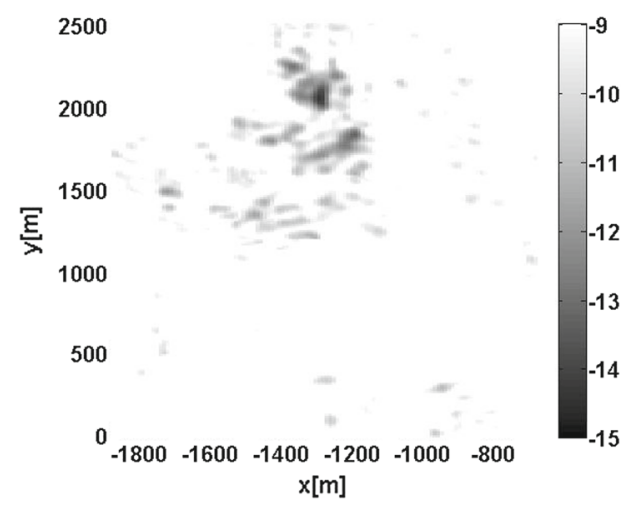

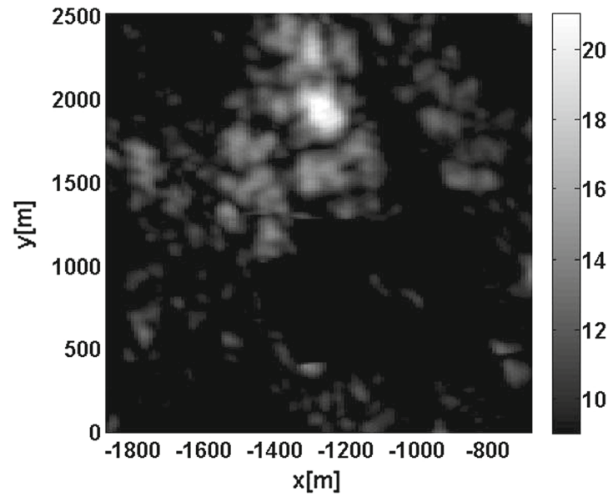
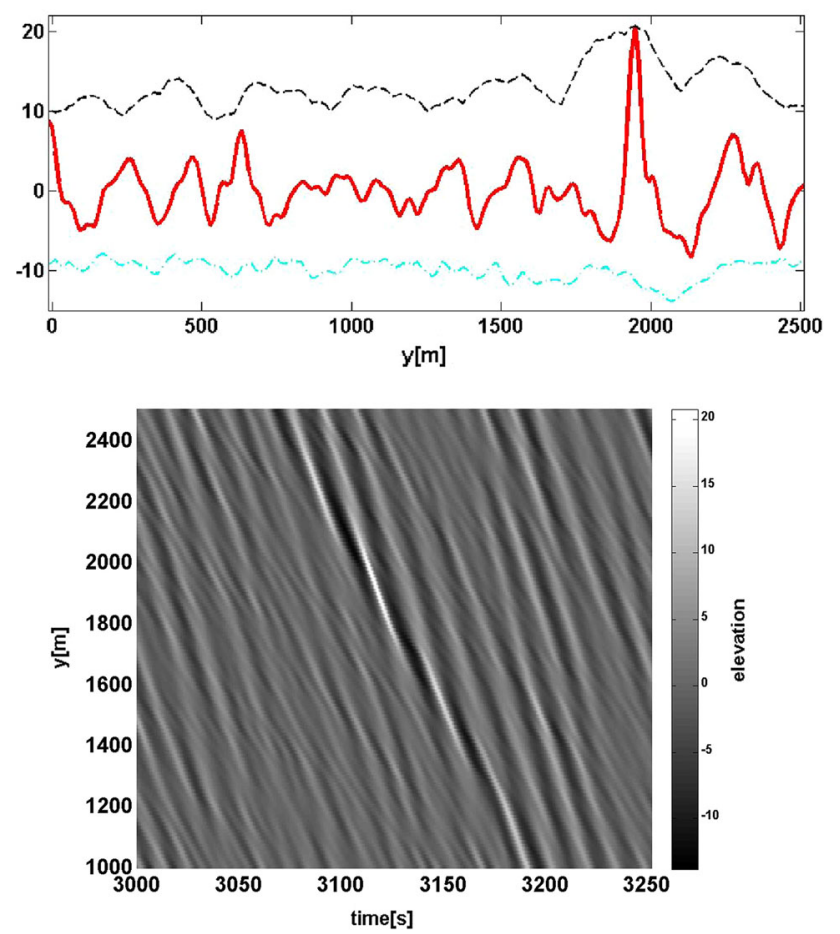

Fig. 18 Upper plot shows the elevation at the time of highest crest in North-South direction for decreasing $y$. The lower plot shows the elevation from North to South vertically downwards, as function of time horizontally in Fig. 4 shows, however, variations of the order of $10 \%$ near the origin. This inhomogeneity indicates that it has been only partly successful to obtain a uniform area; effects of this or other inhomogeneities were not observed.

With regard to numerical accuracy, it should be recognised that a difference of one grid size or time step, caused for instance by small phase errors, may result in a local error in elevation that, in particular for high crests of steep waves, may be considerably more than $1 \mathrm{~m}$. Such errors seem to be unavoidable in simulations as done here. However, a systematic bias has not been found in the area where the waves were analysed and hence the statistical exceedance data will not have been influenced substantially. Therefore, our simulation results provide a reliable insight in the qualitative and quantitative behaviour of Draupner seas for which influence of wind and bottom friction were not taken into account.

As stated before, in our simulations, no wave-breaking takes place for reasonable values of the kinematic breaking criterion, despite the fact that near the highest waves, the steepness can be as large as 0.7 . The elevation exceedance, and the derived crest probability, makes it possible to discuss the encounter probability. As an example, a 'Draupner event', defined as a crest height exceeding $1.5 H_{\mathrm{s}}$, has probability $1.4 \mathrm{e}^{-6}$ per period per gridpoint, and will occur in a time interval of 20 minutes in an area of approximately $900 \times 900$ 
$\mathrm{m}^{2}$, a bit larger than the area of $800 \times 800 \mathrm{~m}^{2}$ as stated in Cavaleri et al. (2016).

The Draupner seas that have been designed, simulated, and characterized show that linear interference arguments can be used to describe the, sometimes confused, nonlinear sea states in a qualitative way. The wide spreading can explain that waves from different directions can occasionally contribute in a coherent way to form isolated, exceptionally high waves with time signals that have the same character as that of the measured Draupner sea. Nonlinear effects are very clearly present in the formation and probability of high crests.

Superposition of oblique waves are essential to form the high crests of the freak waves, as has already been shown by Adcock et al. (2011). The plot of the elevation in Fig. 16 is an illustration of the multi-directionality: with no waves in a large surrounding that are higher than $12 \mathrm{~m}$, an extreme wave larger than $20 \mathrm{~m}$ suddenly arose from waves with different directions, as also noticed from the gradient plot in the same figure.

Acknowledgements We greatly appreciate the help of Peter Janssen from the European Centre for Medium-Range Weather Forecasts, Reading, UK, for sharing the data of the Draupner spectrum. We thank the reviewers of a previous draft for their remarks that led to a substantial improvement of the presentation of the results. Technical assistance from Fany Wresti Buana Putri and Riam Badriana at Labmath-Indonesia in dealing with the ensemble data is gratefully acknowledged. R.K. was partly funded by the Netherlands Organization for Scientific Research NWO, Technical Science Division STW, Project 11642.

Open Access This article is distributed under the terms of the Creative Commons Attribution 4.0 International License (http://creativecomm ons.org/licenses/by/4.0/), which permits unrestricted use, distribution, and reproduction in any medium, provided you give appropriate credit to the original author(s) and the source, provide a link to the Creative Commons license, and indicate if changes were made.

\section{References}

Adcock TAA, Taylor PH, Yan S, Ma QW, Janssen PAEM (2011) Did the Draupner wave occur in a crossing sea? Proc R Soc A 467:30043021

Adcock TAA, Taylor PH, Draper S (2015) Nonlinear dynamics of wavegroups in random seas: unexpected walls of water in the open ocean. Proc R Soc A 471

Broer LJF (1974) On the Hamiltonian theory of surfacewaves. Appl Sci Res 29:430-446

Cavaleri L, Barbariol F, Benetazzo A, Bertotti L, Bidlot J-R, Janssen P, Wedi N (2016) The Draupner wave: a fresh look and the emerging view. J Geophys Res Oceans 121
Cavaleri L, Benetazzo A, Barbariol F, Bidlot JR, Janssen PAEM (2016) The Draupner event: the large wave and the emerging view. Bull Am Meteorol Soc. doi:10.1175/BAMS-D-15-00300.1

Dommermuth DG, Yue DKP (1987) A high-order spectral method for the study of nonlinear gravity waves. J Fluid Mech 184:267

Dysthe K, Krogstad HE, Müller P (2008) Oceanic rogue waves. Annu Rev Fluid Mech 40:287-310

Goda Y (2010) Random seas and design of maritime structures. 3rd edn. World Scientific, Singapore

Haver S (1995) A possible freak wave event measured at the Draupner Jacket January 1 1995. Available at http://www.ifremer.fr/ web-com/stw2004/rogue/fullpapers/walk_on_haver.pdf

HAWASSI software. see http://hawassi.labmath-indonesia.org/

Jefferys E (1987) Directional seas should be ergodic. Appl Ocean Res 9:186-191

Kharif C, Pelinovsky E (2003) Physical mechanisms of the rogue wave phenomenon. Eur J Mech B Fluids 22:603-634

Kharif C, Pelinovsky E, Slunyaev A (2009) Rogue waves in the Ocean. Springer

Kennedy AB, Chen Q, Kirby JT, Dalrymple RA (2000) Boussinesq modeling of wave transformation, breaking, and runup. J Waterw Port Coast Ocean Eng 126:39-47

Korotkevich A, Zakharov VE (2015) Evaluation of a spectral line width for the Phillips spectrum by means of numerical simulation. Nonlin Process Geophys 22:325-335

Kurnia R, Van Groesen E (2014) High order Hamiltonian water wave models with wave-breaking mechanism. Coast Eng 93:55-70

Kurnia R, Van Groesen E (2015) Spatial-spectral Hamiltonian Boussinesq wave simulations. Advances in computational and experimental marine hydrodynamics. Conf Proc 2:19-24

Kurnia R, Van Groesen E (2017) Localization for spatial-spectral implementations of 1D Analytic Boussinesq equations. Wave Motion 72:113-132

Latheef M, Swan C (2013) A laboratory study of wave crest statistics and the role of directional spreading. Proc R Soc A 469:20120696

Lie SL, Adytia D, van Groesen E (2014) Embedded wave generation for dispersive surface wave models. Ocean Eng 80:73-83

Seyffert HC, Kim Dae-Hyun, Troesch AW (2016) Rare wave groups. Ocean Eng 122:241-252

Van Groesen E, Wijaya AP (2017) High waves in Draupner seas, Part 2: Observation and prediction from synthetic radar images. J Ocean Eng Mar Energy. doi:10.1007/s40722-017-0090-x

West BJ, Brueckner KA, Janda RS, Milder DM, Milton RL (1987) A new numerical method for surface hydrodynamics. J Geophys Res 92:11803

Zakharov VE (1968) Stability of periodic waves of finite amplitude on the surface of a deep fluid. J Appl Mech Tech Phys 9:190-194 